\title{
ON HOMOCLINIC POINTS
}

\author{
S. NEWHOUSE
}

\begin{abstract}
Results of R. C. Robinson and D. Pixton on the existence of homoclinic points for diffeomorphisms on the two-sphere are extended. An application to area preserving diffeomorphisms on surfaces is given.
\end{abstract}

The purpose of this note is to extend results of Robinson [7] and Pixton [5] concerning the existence of homoclinic points for diffeomorphisms on two-dimensional manifolds.

The basic problem is this. Suppose $y \in \mathrm{Cl} W^{u}(p, f) \cap\left(W^{s}(p, f)-\{p\}\right)$ where $p$ is a hyperbolic periodic point of a $C^{r}$ diffeomorphism $f$ of a manifold $r \geqslant 1, W^{u}(p, f)$ is the unstable manifold of $p$ while $W^{s}(p, f)$ is the stable manifold of $p$. Is there a small $C^{r}$ perturbation $g$ of $f$ such that $p$ is a hyperbolic periodic point of $g$ and $y \in W^{u}(p, g) \cap W^{s}(p, g)$ ? Following Poincaré, such a point $y$ in $W^{u}(p, g) \cap W^{s}(p, g)$ is called a homoclinic point for $g$. We will also say that $y$ is $(p, g)$-homoclinic. Homoclinic points generally yield interesting phenomena. In particular, as Smale realized [8], [2, Appendix], they usually give the existence of infinitely many periodic points.

From [5] and [7], the above question has a positive answer on the twosphere if $W^{u}(p, f) \cap W^{s}(p, f)=\varnothing$. Here we shall consider any two-dimensional manifold $M$ and a $C^{r}$ diffeomorphism $f: M \rightarrow M$ having a hyperbolic periodic saddle point $p$. We use the Whitney $C^{r}$ topology for perturbations of $f$. Assume that $W^{u}(p, f)$ and $W^{s}(p, f)$ already have a nonempty transversal intersection, say $y_{1}$. Let $W_{1}^{u}(p, f)$ be the component of $W^{u}(p, f)-\{p\}$ containing $y_{1}$, and let $W_{1}^{s}(p, f)$ be the component of $W^{s}(p, f)-\{p\}$ contining $y_{1}$. We wish to take another point $y$ in $W_{1}^{s}(p, f)$ and give a sufficient condition for $y$ to become $(p, g)$-homoclinic for a small $C^{r}$ perturbation $g$ of $f$. Let $W_{0}^{u}(p, f)$ be the component of $W^{u}(p, f)-\{p\}$ not meeting $W_{1}^{u}(p, f)$.

Let $\Omega(f)$ denote the nonwandering set of $f$ and let $\alpha(y, f)$ denote the $\alpha$-limit set of $y$. We recall that $x \in \Omega(f)$ if and only if there are sequences $x_{i} \rightarrow x$ and $n_{i} \rightarrow \infty$ with $f^{n_{i}}\left(x_{i}\right) \rightarrow x$ as $i \rightarrow \infty$ while $x \in \alpha(y, f)$ if and only if there is a sequence $n_{i} \rightarrow-\infty$ with $f^{n_{i}}(y) \rightarrow x$ as $i \rightarrow \infty$.

THEOREM 1. With the above notations, assume $y$ is in $\Omega(f), p$ is not in $\alpha(y, f)$, and $W_{0}^{u}(p, f)$ has some nonempty transversal intersection with $W^{s}(q, f)$ for

Received by the editors November 1, 1975.

AMS (MOS) subject classifications (1970). Primary 34-XX, 34C35.

Key words and phrases. Homoclinic, diffeomorphism, hyperbolic, area preserving. 
some hyperbolic periodic point $q$ of $f$. Then $f$ may be $C^{r}$ perturbed to $g$ so that $y$ is $(p, g)$-homoclinic.

Proof. Let $S$ be a compact arc in $W^{s}(p, f)$ containing $p$ and $y_{1}$ in its interior. Choose a small arc $V_{1}$ in $W_{1}^{u}(p, f)$ such that $y_{1}$ is in its interior and $V_{1} \cap S=\left\{y_{1}\right\}$. Let $n_{0}$ be the least integer so that $f^{n_{0}}(p)=p$. Let $V_{2}$ be a small arc in $W^{s}(q, f)$ having a nonempty transversal intersection with $W_{0}^{u}(p, f)$ in its interior. Observe that $q$ must be a saddle point or a sink. From the $\lambda$-lemma [4], there are arcs in

$$
S^{\prime}=\bigcup_{k<0} f^{k n_{0}}(S) \text { and } V_{2}^{\prime}=\bigcup_{k<0} f^{k n_{0}}\left(V_{2}\right)
$$

which are uniformly $C^{1}$ close to $S$. Hence, replacing $y_{1}$ by another element of its orbit if necessary, we may find a box-like closed neighborhood $N$ of $y$ whose boundary $\partial N=N^{u} \cup N^{s}$ where $N^{u}$ consists of an arc in $V_{1}$ and an arc in $f^{n_{0}} V_{1}$ while $N^{s}$ consists of an arc in $S^{\prime}$ and an arc in $V_{2}^{\prime}$.

The following figure describes $N$ and $\partial N$.

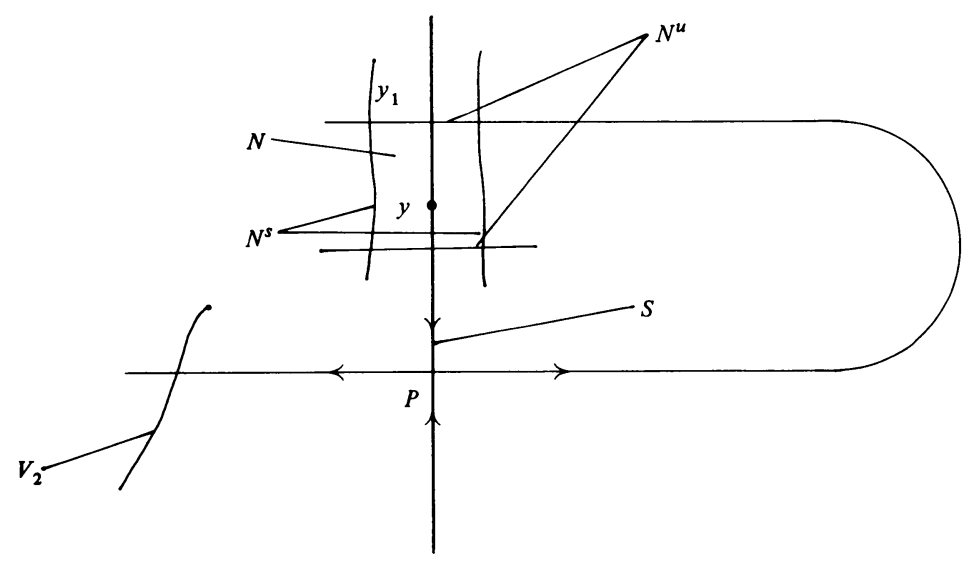

Since $p \notin \alpha(y, f)$, we have that $S \cap \alpha(y, f)=\varnothing$ by the invariance of $\alpha(y, f)$. Since $\alpha(y, f)$ is closed we have that $\alpha(y, f) \cap N=\varnothing$ provided the arcs in $N^{s}$ are close enough to $S$. Choosing these arcs even closer we may arrange that $f^{-n}(y) \notin N$ for all $n>0$ and $f^{-n}\left(N^{u}\right) \cap$ int $N=\varnothing$ for all $n \geqslant 0$.

For $z \in M$, let $o_{-}(z, f)=\left\{f^{-n}(z): n>0\right\}$, and let $o(z)=\left\{f^{n}(z): n=0\right.$, $\pm 1, \pm 2, \ldots\}$. We claim

(1) there is a sequence $x_{i} \in W_{1}^{u}(o(p), f), i=1,2, \ldots$, converging to $y$ so that $o_{-}\left(x_{i}, f\right) \cap$ int $N=\varnothing$ for all large $i$.

Here $W_{1}^{u}(o(p), f)=\cup_{z \in o(p)} W_{1}^{u}(p, f)$.

Once (1) is established, standard methods, as in Robinson [7], enable one to perturb $f$ to $g$ so that $p$ is a hyperbolic saddle periodic point for $g$ and $W^{u}(o(p), g)$ has a transversal intersection with $W^{s}(p, g)$ at $y$. Then it follows from Corollary (1.3) in [4] that $W^{u}(p, g)$ has transversal intersections with $W^{s}(p, g)$ arbitrarily near $y$. In fact, it is known that such points $y_{i}$ may be 
found whose orbits $o\left(y_{i}\right)$ are near $y$ only at $y_{i}$. From this, $g$ may be further perturbed to $g_{1}$ so that $y$ becomes $\left(p, g_{1}\right)$ homoclinic.

We now prove (1). The method is a variant of the one introduced in [7].

For each integer $n \geqslant 0$, let $D_{n}=\cup_{1 \leqslant j \leqslant n} f^{j}(N)$. Since $o_{-}(y, f) \cap N=\varnothing$, we have that $y \notin D_{n}$ for each $n$. Let $x_{n}$ be the point of $D_{n}$ closest to $y$. Clearly,

$$
x_{n} \in \partial D_{n} \subset \bigcup_{1<j<n} \partial\left(f^{j} N\right)=\bigcup_{1<j<n}\left[f^{j}\left(N^{u}\right) \cup f^{j}\left(N^{s}\right)\right] .
$$

We may choose a neighborhood $U$ of $y$ so that $f^{n}\left(N^{s}\right) \cap U=\varnothing$ for $n \geqslant 0$, since for $n>0, f^{-n}(y) \notin N$, and $f^{n}\left(N^{s}\right) \cap N=\varnothing$ for $n$ large. Since $y$ is nonwandering for $f$, there are sequences $y_{i} \rightarrow y$ and $n_{i} \rightarrow \infty$ so that $f^{n_{i}}\left(y_{i}\right) \rightarrow y$ as $i \rightarrow \infty$. Thus, for $i$ large, $\left\{y_{i}, f^{n_{i}}\left(y_{i}\right)\right\} \subset N$. Hence $f^{n_{i}}(N)$ accumulates on $y$, so $x_{n_{i}} \rightarrow y$ as $i \rightarrow \infty$. Let $n_{1}>0$ be such that for $i \geqslant n_{1}, x_{n_{i}} \in U$. Then $x_{n_{i}} \in \cup_{1 \leqslant j \leqslant n_{i}} f^{j}\left(N^{u}\right) \subset W_{1}^{u}(o(p), f)$.

Suppose $o_{-}\left(x_{n_{i}}, f\right) \cap$ int $N \neq \varnothing$ for some $i \geqslant n_{1}$. Then there is an integer $k_{i}>0$ so that $f^{-k_{i}}\left(x_{n_{i}}\right) \in \operatorname{int} N$ or $x_{n_{i}} \in \operatorname{int} f^{k_{i}}(N)$. Since $\cup_{n \geqslant 0} f^{-n}\left(N^{u}\right) \cap$ int $N=\varnothing$, we see that $0<k_{i}<n_{i}$. But then $x_{n_{i}} \in f^{k_{i}}$ (int $\left.N\right) \subset$ int $D_{n_{i}}$, which is impossible since $x_{n_{i}} \in \partial D_{n_{i}}$. Thus, for $i \geqslant n_{1}, o_{-}\left(x_{n_{i}}, f\right) \cap$ int $N=\varnothing$, and the proof is completed.

REMARKS 1. Notice that the $\alpha$-limit set condition on $y$ will be fulfilled if $y \in W^{u}\left(q_{1}, f\right)$ for some hyperbolic periodic point $q_{1}$ not in the orbit of $p$.

2. If $y$ actually is a transversal homoclinic point for $(p, f)$ then $W^{u}(y, f)$ is a limit of infinitely many unstable manifolds of different hyperbolic periodic orbits. Thus, $y$ is a limit of points $y_{i}$ in $W^{s}(y, f)$ so that $p \notin \alpha\left(y_{i}, f\right)$. Theorem 1 should be thought of as a sort of converse to this.

There are analogous results when $f$ is area preserving. Indeed, if $M$ has a smooth 2-form $\omega$ with $\omega(p) \neq 0, f^{*} \omega=\omega$, and $\int_{M} \omega<\infty$, then the perturbation $g$ of $f$ in Theorem 1 may be chosen so that $g^{*} \omega=\omega$ as well. For this one uses generating functions as in [1], [3, §2]. Also, in this case, the point $y$ (and all points in $\left.W^{u}(p, f) \cup W^{s}(p, f)\right)$ will automatically be nonwandering, so that hypothesis may be dropped. Moreover, one has the following result.

THEOREM 2. Let $p$ be a hyperbolic periodic point of a diffeomorphism $f$ on an orientable two-dimensional manifold $M$ having a transversal homoclinic point. Suppose there is a smooth 2-form $\omega$ on $M$ with $\omega(p) \neq 0, f^{*} \omega=\omega$, and $\int_{M} \omega<\infty$. Let $q$ be another hyperbolic periodic point of $f$, and let $y \in W^{u}(q)$ $\cap W^{s}(p)$. Then $f$ may be $C^{r}$ perturbed to $g$ so that $g^{*} \omega=\omega$ and $y$ is a limit of $(p, g)$ homoclinic points.

Proof. By [6], we first perturb $f$ to $f_{1}$ so that $y$ is a transversal intersection of $W^{u}\left(q, f_{1}\right)$ and $W^{s}\left(p, f_{1}\right)$. For $f_{1}$ close enough to $f,\left(p, f_{1}\right)$ still has a transversal homoclinic point.

By Smale's homoclinic point theorem [8], [2, Appendix], $p$ is a limit of a sequence of hyperbolic saddle periodic points $p_{i}$ of $f_{1}$ such that $W^{s}\left(p_{i}, f_{1}\right)$ has nonempty transversal intersections with $W^{u}\left(q, f_{1}\right)$, say $y_{i}$, near $y$. Further, it is 
easily seen that the $p_{i}$ 's may be chosen so that both components of $W^{u}\left(p_{i}, f_{1}\right)-\left\{p_{i}\right\}$ meet $W^{s}\left(p_{i}, f_{1}\right)$. Observe that the $y_{i}$ 's are nonwandering points for $i$ large. Indeed, since $\omega(p) \neq 0$, we have $\omega\left(f_{1}^{n}(y)\right) \neq 0$ for $n>0$ large, so $\omega(y) \neq 0$ as $f_{1}^{*} \omega=\omega$. Hence for $i$ large, $\omega\left(y_{i}\right) \neq 0$. For any such $y_{i}$, if $U$ is a small neighborhood of $y_{i}$, we have $\int_{U} \omega>0$. Since $\int_{U_{n>0} f_{i}^{n} U} \omega \leqslant \int_{M} \omega<$ $\infty$, there are integers $0 \leqslant n_{1}<n_{2}$ so that $f_{1}^{n_{1}}(U) \cap f_{1}^{n_{2}}(U) \neq \varnothing$, whence $f_{1}^{n_{2}-n_{1}}(U) \cap U \neq \varnothing$. Thus, $y_{i}$ is nonwandering. By Theorem 1 and the remarks about generating functions preceding the statement of Theorem $2, f_{1}$ may be perturbed to make $y_{i}$ homoclinic, and Theorem 2 is proved.

ReMARK. If $M$ is compact, and $f$ is area preserving, then Poincare expected that generically $W^{u}(p) \cap W^{s}(p)$ would be dense in $W^{u}(p)$ for any hyperbolic periodic point $p$. Takens has proved this in the $C^{1}$ topology [9]. However, the problem remains unsolved in the $C^{r}$ topology, $r \geqslant 2$.

\section{REFERENCES}

1. V. Arnold and A. Avez, Ergodic problems of classical mechanics, Gauthier-Villars, Paris, 1967; English transl., Benjamin, New York, 1968. MR 35 \#334; 38 \# 1233.

2. S. Newhouse, Hyperbolic limit sets, Trans. Amer. Math. Soc. 167 (1972), 125-150. MR 45 \#4454.

3. _ Quasi-elliptic periodic points in conservative dynamical systems, Amer. J. Math. (to appear).

4. J. Palis, On Morse-Smale dynamical systems, Topology 8 (1968), 385-404. MR 39 \#620.

5. D. Pixton, A closing lemma for invariant manifolds, Thesis, Univ. of California, Berkeley, Calif., 1974.

6. R. Clark Robinson, Generic properties of conservative systems, Amer. J. Math. 92 (1970), 562-603. MR 42 \#8517.

7. Clark Robinson, Closing stable and unstable manifolds on the two-sphere, Proc. Amer. Math. Soc. 41 (1973), 299-303. MR 47 \#9674.

8. S. Smale, Diffeomorphisms with many periodic points, Differential and Combinatorial Topology, Princeton Univ. Press, Princeton, N. J., 1965, pp. 63-80. MR 31 \#6244.

9. F. Takens, Homoclinic points in conservative systems, Invent. Math. 18 (1972), 267-292. MR 48 \#9768.

Department of Mathematics, University of North Carolina, Chapel Hill, North Carolina 27514 